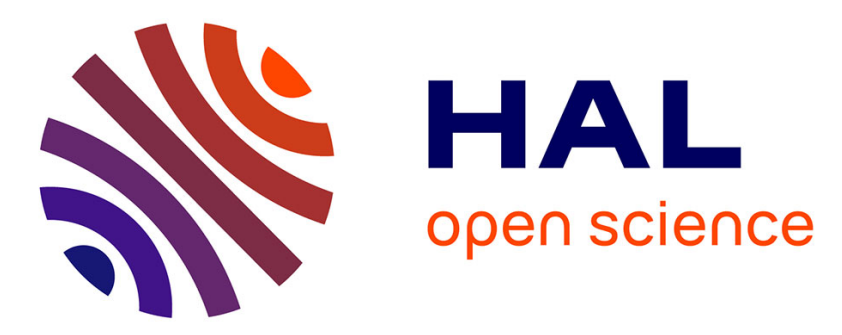

\title{
Convergence of the generalized- $\alpha$ scheme for constrained mechanical systems
}

\author{
Martin Arnold, Olivier Brüls
}

\section{To cite this version:}

Martin Arnold, Olivier Brüls. Convergence of the generalized- $\alpha$ scheme for constrained mechanical systems. Multibody System Dynamics, 2007, 85, pp.187-202. 10.1007/s11044-007-9084-0 . hal01490825

\section{HAL Id: hal-01490825 \\ https://hal.science/hal-01490825}

Submitted on 16 Mar 2017

HAL is a multi-disciplinary open access archive for the deposit and dissemination of scientific research documents, whether they are published or not. The documents may come from teaching and research institutions in France or abroad, or from public or private research centers.
L'archive ouverte pluridisciplinaire HAL, est destinée au dépôt et à la diffusion de documents scientifiques de niveau recherche, publiés ou non, émanant des établissements d'enseignement et de recherche français ou étrangers, des laboratoires publics ou privés. 


\title{
Convergence of the generalized- $\alpha$ scheme for constrained mechanical systems
}

\author{
Martin Arnold • Olivier Brüls
}

\begin{abstract}
A variant of the generalized- $\alpha$ scheme is proposed for constrained mechanical systems represented by index-3 DAEs. Based on the analogy with linear multistep methods, an elegant convergence analysis is developed for this algorithm. Second-order convergence is demonstrated both for the generalized coordinates and the Lagrange multipliers, and those theoretical results are illustrated by numerical tests.
\end{abstract}

Keywords DAEs · Generalized- $\alpha$ method

\section{Introduction}

The generalized- $\alpha$ scheme has been initially developed for the simulation of finite element models in structural dynamics. It allows a simple and efficient implementation, as well as an optimal combination of accuracy at low-frequency and numerical damping at high-frequency. This last feature is especially interesting, since it allows to eliminate the contribution of non-physical high-frequency modes, which are generally present in finite element models. The generalized- $\alpha$ algorithm results from successive contributions by Newmark [18], Hilber, Hughes and Taylor [14], and Chung and Hulbert [9]; an overview of its properties in the non-linear regime is also given by Erlicher et al. [10].

This work concerns the employment of the generalized- $\alpha$ scheme for the simulation of constrained mechanical systems. Cardona and Géradin [8] have shown that numerical damping is critical to avoid numerical oscillations in the Lagrange multipliers. Their theoretical investigations are restricted to linear problems, but they also report consistent results in nonlinear test cases. However, it is well-known that the order of an integration algorithm can

\footnotetext{
M. Arnold (凶)

NWF III-Institute of Mathematics, Martin Luther University Halle-Wittenberg, 06099 Halle (Saale), Germany

e-mail: martin.arnold@mathematik.uni-halle.de

O. Brüls

Department of Aerospace and Mechanical Engineering, University of Liège, 4000 Liège, Belgium e-mail: o.bruls@ulg.ac.be
} 
be reduced due to the presence of algebraic constraints [4, 13], which calls for a rigorous convergence analysis of the generalized- $\alpha$ method in the context of differential-algebraic systems. Recently, Lunk and Simeon [16] and Jay and Negrut [15] have proven secondorder convergence for regularized index-2 formulations, i.e. algorithms which make use of kinematic constraints at both position and velocity level.

In contrast, an algorithm based on an index-3 formulation, i.e., solely based on position level constraints, is analysed in this paper. This algorithm can deal with a non-constant mass matrix and it computes the acceleration variables with second-order accuracy. It extends the approach of Negrut et al. [17] who report on positive practical experience with the HilberHughes-Taylor algorithm applied to the index-3 formulation of the equations of motion in an industrial multibody system simulation tool. Recently, Bottasso et al. [3] proposed a scaling technique to reduce the numerical instability effects being typical of such time integration methods for DAEs of index 3, see [1, 2, 4].

In the present paper, the analogy between generalized- $\alpha$ algorithms and linear multistep integrators helps to get a simpler and more intuitive convergence proof, see also the rather technical work of Lunk and Simeon [16] or Jay and Negrut [15]. Second-order convergence is demonstrated for both the generalized coordinates, and the Lagrange multipliers. The remaining part of the paper is organized as follows: the generalized- $\alpha$ algorithm and its multistep representation are introduced in Sect. 2. Estimates for local and global errors are proven in Sect. 3. Some technical parts of the proof are collected separately in Sect. 4. In Sect. 5, the results of the theoretical investigations are illustrated by numerical tests. The conclusions in Sect. 6 summarize essential parts of the paper.

\section{The generalized- $\alpha$ method}

Let us consider the constrained mechanical system

$$
\begin{aligned}
& \mathbf{M}(\mathbf{q}) \ddot{\mathbf{q}}=\mathbf{f}(\mathbf{q}, \dot{\mathbf{q}}, t)-\boldsymbol{\Phi}_{\mathbf{q}}^{T} \lambda, \\
& \mathbf{0}=\boldsymbol{\Phi}(\mathbf{q}, t)
\end{aligned}
$$

where (1) represents the dynamics of the mechanical system and (2) represents the kinematic constraints. The vectors $\mathbf{q}$ and $\lambda$ denote the generalized coordinates and the Lagrange multipliers, respectively, $\mathbf{M}$ is the symmetric mass matrix, the vector of apparent forces $\mathbf{f}$ collects external forces, internal forces and complementary inertia forces, and $\boldsymbol{\Phi}_{\mathbf{q}}$ is the matrix of constraint gradients. The mass matrix is not necessarily constant, but it may depend on the generalized coordinates $\mathbf{q}$, which allows to cover the case of mechanical systems with large rotations. We note that the present developments could be further extended to mechatronic problems, as suggested by Bruls and Golinval [6, 7].

\subsection{Description of the algorithm}

We propose an implementation of the generalized- $\alpha$ method which does not rely on a weighted formulation of the residual equation. Instead, the dynamic equilibrium is enforced exactly at every time step, with three major advantages: (i) the accelerations are computed with second-order accuracy, (ii) the consistency of the algorithm is not affected if the mass matrix is not constant, and (iii) the algorithm is closer to the physics of the problem, which also simplifies theoretical investigations. 
Hence, the numerical variables $\mathbf{q}_{n+1}, \dot{\mathbf{q}}_{n+1}, \ddot{\mathbf{q}}_{n+1}, \boldsymbol{\lambda}_{n+1}$ satisfy (1) and (2) at time $t=t_{n+1}$, whereas, the vector a of acceleration-like variables is defined by the recurrence relation

$$
\left(1-\alpha_{m}\right) \mathbf{a}_{n+1}+\alpha_{m} \mathbf{a}_{n}=\left(1-\alpha_{f}\right) \ddot{\mathbf{q}}_{n+1}+\alpha_{f} \ddot{\mathbf{q}}_{n}, \quad \mathbf{a}_{0}=\ddot{\mathbf{q}}_{0} .
$$

We emphasize that $\mathbf{a}$ is an auxiliary variable, which is not equal to the true accelerations $\ddot{\mathbf{q}}$. Since $\mathbf{M}$ depends on $\mathbf{q}$, this equation cannot be restated as a weighted form of (1). The generalized- $\alpha$ scheme is obtained using a in the Newmark integration formulae

$$
\begin{aligned}
& \mathbf{q}_{n+1}=\mathbf{q}_{n}+h \dot{\mathbf{q}}_{n}+h^{2}\left(\frac{1}{2}-\beta\right) \mathbf{a}_{n}+h^{2} \beta \mathbf{a}_{n+1}, \\
& \dot{\mathbf{q}}_{n+1}=\dot{\mathbf{q}}_{n}+h(1-\gamma) \mathbf{a}_{n}+h \gamma \mathbf{a}_{n+1}
\end{aligned}
$$

where $h$ is the step-size. The numerical parameters $\alpha_{m}, \alpha_{f}, \beta$ and $\gamma$ can be selected in order to have suitable accuracy and stability properties.

Algorithm 1 solves formulae (3), (4), and (5) together with the dynamic equilibrium at time $t_{n+1}$. The correction step involves the parameters

$$
\beta^{\prime}=\frac{1-\alpha_{m}}{h^{2} \beta\left(1-\alpha_{f}\right)}, \quad \gamma^{\prime}=\frac{\gamma}{h \beta}
$$

which satisfy the properties

$$
\frac{\partial \ddot{\mathbf{q}}_{n+1}}{\partial \mathbf{q}_{n+1}}=\mathbf{I} \beta^{\prime}, \quad \frac{\partial \dot{\mathbf{q}}_{n+1}}{\partial \mathbf{q}_{n+1}}=\mathbf{I} \gamma^{\prime} .
$$

Moreover, the iteration matrix is given by

$$
\mathbf{S}_{t}=\left[\begin{array}{cc}
\left(\mathbf{M} \beta^{\prime}+\mathbf{C}_{t} \gamma^{\prime}+\mathbf{K}_{t}\right) & \boldsymbol{\Phi}_{\mathbf{q}}^{T} \\
\boldsymbol{\Phi}_{\mathbf{q}} & \mathbf{0}
\end{array}\right]
$$

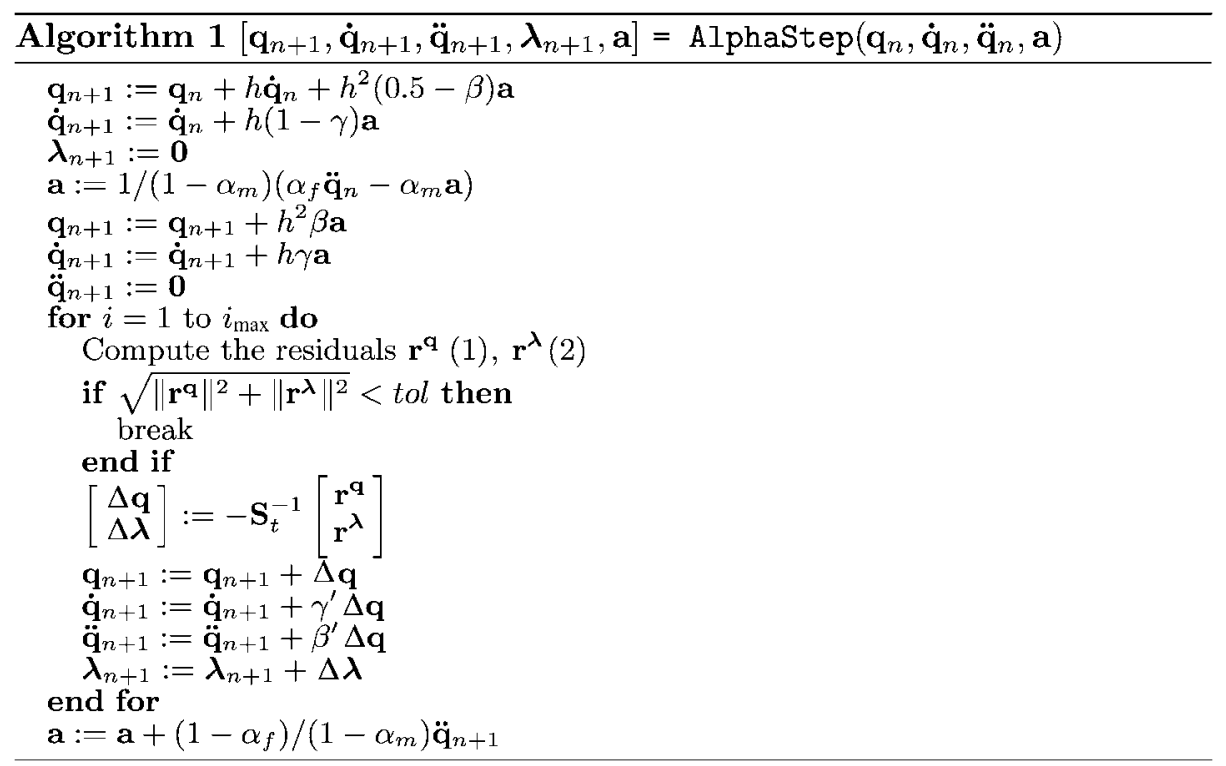


with the tangent stiffness matrix $\mathbf{K}_{t}=\partial\left(\mathbf{M} \ddot{\mathbf{q}}-\mathbf{f}+\boldsymbol{\Phi}_{\mathbf{q}}^{T} \lambda\right) / \partial \mathbf{q}$ and the tangent damping matrix $\mathbf{C}_{t}=\partial(-\mathbf{f}) / \partial \dot{\mathbf{q}}$. Compared to a classical algorithm based on a weighted formulation of (1), as described in [9, 14], Algorithm 1 involves similar computational resources. Indeed, it only requires one additional vector a, and the correction step, which is the most demanding part of the algorithm, is barely modified.

For small time steps $h$, the matrix $\mathbf{S}_{t}$ becomes severely ill conditioned. Bottasso et al. [3] have proposed a scaling method in order to avoid this phenomenon, see also the closely related scaling approach of Hairer and Wanner [13] in the classical general purpose DAE solver RADAU5. The linear system

$$
\mathbf{S}_{t} \mathbf{x}=-\mathbf{r},
$$

with $\mathbf{x}^{T}=\left[\Delta \mathbf{q}^{T} \Delta \lambda^{T}\right]$ and $\mathbf{r}^{T}=\left[\left(\mathbf{r}^{\mathbf{q}}\right)^{T}\left(\mathbf{r}^{\lambda}\right)^{T}\right]$ is replaced by the equivalent scaled form

$$
\overline{\mathbf{S}}_{t} \overline{\mathbf{x}}=-\overline{\mathbf{r}}
$$

with $\overline{\mathbf{S}}_{t}=\mathbf{D}_{L} \mathbf{S}_{t} \mathbf{D}_{R}, \overline{\mathbf{x}}=\mathbf{D}_{R}^{-1} \mathbf{x}$ and $\overline{\mathbf{r}}=\mathbf{D}_{L} \mathbf{r}$. In the numerical tests presented in Sect. 5, the diagonal left and right preconditioners

$$
\mathbf{D}_{L}=\left[\begin{array}{cc}
\mathbf{I} \beta h^{2} & \mathbf{0} \\
\mathbf{0} & \mathbf{I}
\end{array}\right], \quad \mathbf{D}_{R}=\left[\begin{array}{cc}
\mathbf{I} & \mathbf{0} \\
\mathbf{0} & \mathbf{I} / \beta h^{2}
\end{array}\right]
$$

are used to achieve an optimal conditioning of the matrix $\overline{\mathbf{S}}_{t}$, see [3].

\subsection{Multistep representation of the algorithm}

Assuming that the mass matrix is non-singular, the dynamic equilibrium is equivalent to an explicit form

$$
\begin{aligned}
\ddot{\mathbf{q}} & =\mathbf{g}(\mathbf{q}, \dot{\mathbf{q}}, \lambda, t), \\
\mathbf{0} & =\boldsymbol{\Phi}(\mathbf{q}, t)
\end{aligned}
$$

with $\mathbf{g}=\mathbf{M}^{-1}\left(\mathbf{f}-\boldsymbol{\Phi}_{\mathbf{q}}^{T} \lambda\right)$. We also assume that the constrained system $(11,12)$ has DAE index 3, i.e., that the matrix

$$
\Phi_{\mathrm{q}} \mathbf{g}_{\lambda}
$$

is non-singular, where $\mathbf{g}_{\lambda}$ is given by $\mathbf{g}_{\lambda}=-\mathbf{M}^{-1} \boldsymbol{\Phi}_{\mathbf{q}}^{T}$. Since the dynamic equilibrium is enforced at every time step, the algorithm leads to the same solution when applied to the system $(11,12)$, and it is sufficient to analyse this equivalent system.

It is possible to eliminate a from the integration formulae at time steps $t_{n-1} \rightarrow t_{n}$ and $t_{n} \rightarrow t_{n+1}$, leading to a two-step formulation [10]

$$
\begin{aligned}
& \sum_{k=0}^{2} a_{k} \mathbf{q}_{n+k-1}+h \sum_{k=0}^{1} u_{k} \dot{\mathbf{q}}_{n+k-1}=h^{2} \sum_{k=0}^{2} b_{k} \mathbf{g}_{n+k-1}, \\
& \sum_{k=0}^{2} a_{k} \dot{\mathbf{q}}_{n+k-1}=h \sum_{k=0}^{2} c_{k} \mathbf{g}_{n+k-1}, \\
& \mathbf{0}=\boldsymbol{\Phi}\left(\mathbf{q}_{n+1}, t_{n+1}\right)
\end{aligned}
$$


with the coefficients

$$
\begin{array}{lll}
a_{0}=-\alpha_{m}, & a_{1}=-1+2 \alpha_{m}, & a_{2}=1-\alpha_{m}, \\
u_{0}=-\alpha_{m}, & u_{1}=-1+\alpha_{m}, & \\
b_{0}=\alpha_{f}(1 / 2-\beta), & b_{1}=\left(1-\alpha_{f}\right)(1 / 2-\beta)+\alpha_{f} \beta, & b_{2}=\left(1-\alpha_{f}\right) \beta, \\
c_{0}=\alpha_{f}(1-\gamma), & c_{1}=\left(1-\alpha_{f}\right)(1-\gamma)+\alpha_{f} \gamma, & c_{2}=\left(1-\alpha_{f}\right) \gamma .
\end{array}
$$

In Sect. 3 below, we will see that the detailed analysis of the error propagation in the difference quotients

$$
\mathbf{q}_{n}^{\prime}:=\frac{\mathbf{q}_{n}-\mathbf{q}_{n-1}}{h} \approx \dot{\mathbf{q}}\left(t_{n}-\frac{h}{2}\right)
$$

is very useful to study the error propagation for the Lagrange multipliers $\lambda$. A two-step recursion for $\mathbf{q}^{\prime}$ is obtained by the difference quotient of (14) in its original form and (14) with $n$ being substituted by $n-1$. In this difference quotient, the velocities $\dot{\mathbf{q}}$ may be eliminated using (15) resulting finally in the multistep formula

$$
\sum_{k=0}^{2} a_{k} \mathbf{q}_{n+k-1}^{\prime}=h \sum_{k=0}^{3} b_{k}^{\prime} \mathbf{g}_{n+k-2}
$$

with coefficients

$$
\begin{aligned}
& b_{0}^{\prime}=\alpha_{f}(1 / 2+\beta-\gamma), \\
& b_{1}^{\prime}=\left(1-\alpha_{f}\right)(1 / 2+\beta-\gamma)+\alpha_{f}(1 / 2-2 \beta+\gamma), \\
& b_{2}^{\prime}=\left(1-\alpha_{f}\right)(1 / 2-2 \beta+\gamma)+\alpha_{f} \beta, \\
& b_{3}^{\prime}=\left(1-\alpha_{f}\right) \beta .
\end{aligned}
$$

\subsection{Choice of the numerical parameters}

In the present paper, generalized- $\alpha$ algorithms with fixed step-sizes $h$ are considered. In that case, the generalized- $\alpha$ algorithm for unconstrained mechanical systems is second-order accurate provided that [9]

$$
\gamma=\frac{1}{2}+\alpha_{f}-\alpha_{m}
$$

Note, however, that this condition is no more valid for variable step-size algorithms and should be replaced by an update condition for the parameter $\gamma$, which means that the value of $\gamma$ should be adapted at each time step to guarantee second-order accuracy [5].

The numerical solution is zero-stable (i.e. stable for $h \rightarrow 0$ ) if the polynomial

$$
\varrho(\zeta):=\sum_{k=0}^{2} a_{k} \zeta^{k}
$$

satisfies the root condition, i.e., if condition $\left|\zeta_{i}\right| \leq 1$ is satisfied for all roots $\zeta_{i}, i=1,2$ of polynomial $\varrho$. For multiple roots $\zeta_{i}$, the stronger condition $\left|\zeta_{i}\right|<1$ has to be enforced [12]. Since the roots of $\varrho$ are $\zeta_{1}=1$ and $\zeta_{2}=-\alpha_{m} /\left(1-\alpha_{m}\right)$, zero stability requires $\alpha_{m} \leq 0.5$. We note that both roots are necessarily simple. 
The algorithm is strictly stable at infinity (i.e. strictly stable for $h \rightarrow \infty$, see [13]) if all roots $\zeta_{i}^{\prime}$ of the polynomial

$$
\sigma(\zeta):=\sum_{k=0}^{3} b_{k}^{\prime} \zeta^{k}
$$

satisfy $\left|\zeta_{i}^{\prime}\right|<1, i=1,2,3$. Lemma 1 (see Sect. 4 below) shows that strict stability at infinity is guaranteed if

$$
\alpha_{m}<\alpha_{f}<\frac{1}{2}, \quad \beta>\frac{1}{4}+\frac{1}{2}\left(\alpha_{f}-\alpha_{m}\right)
$$

and the order 2 condition (20) is satisfied.

For stiff problems, the numerical solution should be computed accurately only in the low-frequency range, whereas the high-frequency response should rather be damped out by the algorithm. The high-frequency numerical damping is represented by the spectral radius of the algorithm at infinity $\rho_{\infty}$ : An undamped scheme is characterized by $\rho_{\infty}=1$, whereas $\rho_{\infty}=0$ means asymptotic annihilation of the high-frequency response. For a given value of $\rho_{\infty} \in[0,1]$, Chung and Hulbert [9] have proposed optimal algorithmic parameters for second-order ODEs

$$
\alpha_{m}=\frac{2 \rho_{\infty}-1}{\rho_{\infty}+1}, \quad \alpha_{f}=\frac{\rho_{\infty}}{\rho_{\infty}+1}, \quad \beta=\frac{1}{4}\left(\gamma+\frac{1}{2}\right)^{2}
$$

whereas $\gamma$ is computed according to (20). For $\rho_{\infty} \in[0,1)$, the resulting algorithms are both zero-stable and strictly stable at infinity. Indeed, $\rho_{\infty}<1$ implies

$$
\alpha_{m}<\alpha_{f}<\frac{1}{2}, \quad \gamma>\frac{1}{2}
$$

and

$$
\beta=\frac{1}{4}\left(\gamma+\frac{1}{2}\right)^{2}>\frac{1}{4}\left(\gamma+\frac{1}{2}\right)^{2}-\frac{1}{4}\left(\gamma-\frac{1}{2}\right)^{2}=\frac{1}{2} \gamma=\frac{1}{4}+\frac{1}{2}\left(\alpha_{f}-\alpha_{m}\right),
$$

so that both conditions in (23) are satisfied.

\section{Convergence analysis}

\subsection{Local truncation error}

By definition, the method is convergent of order 2 in the classical unconstrained case if $\left\|\mathbf{q}\left(t_{n}\right)-\mathbf{q}_{n}\right\|$ and $\left\|\dot{\mathbf{q}}\left(t_{n}\right)-\dot{\mathbf{q}}_{n}\right\|$ are $\mathcal{O}\left(h^{2}\right)$. In contrast, the order 2 condition (20) means that the local error, i.e., the error after one time step, is $\mathcal{O}\left(h^{3}\right)$. It can be demonstrated [12] that this last condition is satisfied if $\mathbf{l}_{n}^{\mathbf{q}}$ and $\mathbf{l}_{n}^{\dot{\mathbf{q}}}$ are $\mathcal{O}\left(h^{3}\right)$, where the local truncation errors $\mathbf{l}_{n}^{\mathbf{q}}$ and $\mathbf{l}_{n}^{\dot{\mathbf{q}}}$ are defined by introducing the exact solution $\mathbf{q}\left(t_{n}\right), \dot{\mathbf{q}}\left(t_{n}\right), \lambda\left(t_{n}\right)$ into (14) and (15)

$$
\begin{aligned}
& \mathbf{l}_{n}^{\mathbf{q}}=\sum_{k=0}^{2} a_{k} \mathbf{q}\left(t_{n+k-1}\right)+h \sum_{k=0}^{1} u_{k} \dot{\mathbf{q}}\left(t_{n+k-1}\right)-h^{2} \sum_{k=0}^{2} b_{k} \mathbf{g}\left(t_{n+k-1}\right), \\
& \mathbf{l}_{n}^{\dot{\mathbf{q}}}=\sum_{k=0}^{2} a_{k} \dot{\mathbf{q}}\left(t_{n+k-1}\right)-h \sum_{k=0}^{2} c_{k} \mathbf{g}\left(t_{n+k-1}\right)
\end{aligned}
$$

with the notation $\mathbf{g}\left(t_{n}\right)=\mathbf{g}\left(\mathbf{q}\left(t_{n}\right), \dot{\mathbf{q}}\left(t_{n}\right), \lambda\left(t_{n}\right), t_{n}\right)$. 
For the extension of these results to the constrained case, see $(11,12)$, we consider furthermore, the local truncation error $\mathbf{l}_{n}^{\mathbf{q}^{\prime}}$ in (18)

$$
\mathbf{l}_{n}^{\mathbf{q}^{\prime}}=\sum_{k=0}^{2} a_{k} \frac{\mathbf{q}\left(t_{n+k-1}\right)-\mathbf{q}\left(t_{n+k-2}\right)}{h}-h \sum_{k=0}^{3} b_{k}^{\prime} \mathbf{g}\left(t_{n+k-2}\right) .
$$

The classical order 2 condition in (20) implies that the new consistency conditions

$$
\sum_{k=0}^{2} a_{k}=0 \quad \text { and } \quad \frac{1}{(j+1) !} \sum_{k=0}^{2} a_{k}\left((k-1)^{j+1}-(k-2)^{j+1}\right)=\frac{1}{(j-1) !} \sum_{k=0}^{3} b_{k}^{\prime}(k-2)^{j-1}
$$

$(j=1,2)$, are satisfied and the estimate $\left\|\mathbf{l}_{n}^{\mathbf{q}^{\prime}}\right\|=\mathcal{O}\left(h^{3}\right)$ may be shown by Taylor expansion.

\subsection{Error propagation in the differential solution components}

When a zero-stable algorithm is used to solve an ODE, the order condition implies global convergence [12]. The extension of this classical result from the ODE case to the constrained system in $(11,12)$, i.e., to index-3 DAEs, follows the basic lines of the convergence analysis for multistep methods applied to DAEs, see [13].

In a first step, let us analyse the error propagation during the integration process for the differential components $\mathbf{q}$, $\dot{\mathbf{q}}$ and $\mathbf{q}^{\prime}$. Using the multistep formulae (14), (15) and (18), the defect (25), (26) and (27) become

$$
\begin{aligned}
\mathbf{l}_{n}^{\mathbf{q}} & =\sum_{k=0}^{2} a_{k} \mathbf{e}_{n+k-1}^{\mathbf{q}}+h \sum_{k=0}^{1} u_{k} \mathbf{e}_{n+k-1}^{\dot{\mathbf{q}}}-h^{2} \sum_{k=0}^{2} b_{k} \mathbf{e}_{n+k-1}^{\mathbf{g}}, \\
\mathbf{l}_{n}^{\dot{\mathbf{q}}} & =\sum_{k=0}^{2} a_{k} \mathbf{e}_{n+k-1}^{\dot{\mathbf{q}}}-h \sum_{k=0}^{2} c_{k} \mathbf{e}_{n+k-1}^{\mathbf{g}}, \\
\mathbf{I}_{n}^{\mathbf{q}^{\prime}} & =\sum_{k=0}^{2} a_{k} \mathbf{e}_{n+k-1}^{\mathbf{q}^{\prime}}-h \sum_{k=0}^{3} b_{k}^{\prime} \mathbf{e}_{n+k-2}^{\mathbf{g}}
\end{aligned}
$$

where $\mathbf{e}_{n}^{(\bullet)}=(\bullet)\left(t_{n}\right)-(\bullet)_{n}$ represent a global error after $n$ steps and

$$
\mathbf{e}_{n}^{\mathbf{q}^{\prime}}:=\frac{\mathbf{q}\left(t_{n}\right)-\mathbf{q}\left(t_{n-1}\right)}{h}-\frac{\mathbf{q}_{n}-\mathbf{q}_{n-1}}{h}=\frac{\mathbf{e}_{n}^{\mathbf{q}}-\mathbf{e}_{n-1}^{\mathbf{q}}}{h},
$$

see (17). Inspired by the identical structure of the first terms in the right-hand side of (28-30), the three differential components $\mathbf{q}, \dot{\mathbf{q}}$ and $\mathbf{q}^{\prime}$ and the corresponding local and global errors are summarized in

$$
\begin{aligned}
& \mathbf{v}\left(t_{n}\right):=\left(\begin{array}{c}
\mathbf{q}\left(t_{n}\right) \\
\dot{\mathbf{q}}\left(t_{n}\right) \\
\mathbf{q}^{\prime}\left(t_{n}\right)
\end{array}\right), \quad \mathbf{v}_{n}:=\left(\begin{array}{c}
\mathbf{q}_{n} \\
\dot{\mathbf{q}}_{n} \\
\mathbf{q}_{n}^{\prime}
\end{array}\right), \\
& \mathbf{e}_{n}^{\mathbf{v}}:=\left(\begin{array}{c}
\mathbf{e}_{n}^{\mathbf{q}} \\
\mathbf{e}_{n}^{\dot{\mathbf{q}}} \\
\mathbf{e}_{n}^{\mathbf{q}^{\prime}}
\end{array}\right), \quad \mathbf{l}_{n}^{\mathbf{v}}:=\left(\begin{array}{c}
\mathbf{l}_{n}^{\mathbf{q}} \\
\mathbf{l}_{n}^{\dot{\mathbf{q}}} \\
\mathbf{l}_{n}^{\mathbf{q}^{\prime}}
\end{array}\right)=\mathcal{O}\left(h^{3}\right) .
\end{aligned}
$$


With this compact notation, the error recursion defined by (28), (29) and (30) may be written as

$$
\begin{aligned}
\mathbf{e}_{n+1}^{\mathbf{v}} & =-\frac{a_{0}}{a_{2}} \mathbf{e}_{n-1}^{\mathbf{v}}-\frac{a_{1}}{a_{2}} \mathbf{e}_{n}^{\mathbf{v}}+\mathcal{O}(h) \sum_{k=0}^{1}\left\|\mathbf{e}_{n+k-1}^{\dot{\mathbf{q}}}\right\|+\mathcal{O}(h) \sum_{k=0}^{3}\left\|\mathbf{e}_{n+k-2}^{\mathbf{g}}\right\|+\mathcal{O}\left(h^{3}\right) \\
& =-\frac{a_{0}}{a_{2}} \mathbf{e}_{n-1}^{\mathbf{v}}-\frac{a_{1}}{a_{2}} \mathbf{e}_{n}^{\mathbf{v}}+\mathcal{O}(h) \sum_{k=0}^{3}\left(\left\|\mathbf{e}_{n+k-2}^{\mathbf{v}}\right\|+\left\|\mathbf{e}_{n+k-2}^{\lambda}\right\|\right)+\mathcal{O}\left(h^{3}\right) .
\end{aligned}
$$

The propagation of the errors $\mathbf{e}_{n}^{\mathbf{q}}, \mathbf{e}_{n}^{\dot{\mathbf{q}}}$ and $\mathbf{e}_{n}^{\mathbf{q}^{\prime}}$ is dominated by the recursion coefficients $a_{0}, a_{1}$ and $a_{2}$, and it is coupled with the errors $\mathbf{e}_{n}^{\lambda}$ in the algebraic components $\lambda$ by $\mathcal{O}(h)$ coupling coefficients.

\subsection{Error propagation in the algebraic solution components}

For the analysis of the error propagation in the algebraic solution components $\lambda$, we assume that throughout integration the numerical solution $\left(\mathbf{q}_{n}, \dot{\mathbf{q}}_{n}, \lambda_{n}\right)$ remains in a small neighbourhood of the analytical solution $\left(\mathbf{q}\left(t_{n}\right), \dot{\mathbf{q}}\left(t_{n}\right), \lambda\left(t_{n}\right)\right)$

$$
\left\|\mathbf{e}_{n}^{\mathbf{q}}\right\| \leq C h, \quad\left\|\mathbf{e}_{n}^{\dot{\mathbf{q}}}\right\| \leq C h, \quad\left\|\mathbf{e}_{n}^{\lambda}\right\| \leq C h \quad(n \geq 0)
$$

with a constant $C>0$ that is independent of $n$ and $h$.

Because of the $\mathcal{O}\left(h^{2}\right)$ error bounds for $\left\|\mathbf{e}_{n}^{\mathbf{q}}\right\|,\left\|\mathbf{e}_{n}^{\dot{\mathbf{q}}}\right\|,\left\|\mathbf{e}_{n}^{\lambda}\right\|$ in (41) below, the additional assumption in (34) is always satisfied if $h>0$ is sufficiently small and the initial values of the numerical solution are sufficiently close to the analytical solution (see also part (c) of the proof of Theorem VII.3.5 in [13] for a more detailed discussion).

The conditions on $\left\|\mathbf{e}_{n}^{\mathbf{q}}\right\|,\left\|\mathbf{e}_{n}^{\dot{\mathbf{q}}}\right\|,\left\|\mathbf{e}_{n}^{\lambda}\right\|$ in (34) allow to get an estimate for $\sum_{k} b_{k}^{\prime} \mathbf{e}_{n+k-2}^{\lambda}$ from (30). With

$$
\boldsymbol{\psi}(\vartheta):=\mathbf{g}\left(\mathbf{q}_{n+k-2}+\vartheta \mathbf{e}_{n+k-2}^{\mathbf{q}}, \dot{\mathbf{q}}_{n+k-2}+\vartheta \mathbf{e}_{n+k-2}^{\dot{\mathbf{q}}}, \lambda_{n+k-2}+\vartheta \mathbf{e}_{n+k-2}^{\lambda}, t_{n+k-2}\right)
$$

we have

$$
\begin{aligned}
\mathbf{e}_{n+k-2}^{\mathbf{g}}= & \boldsymbol{\psi}(1)-\boldsymbol{\psi}(0)=\int_{0}^{1} \boldsymbol{\psi}^{\prime}(\vartheta) \mathrm{d} \vartheta \\
= & \int_{0}^{1} \mathbf{g}_{\lambda}\left(\mathbf{q}_{n+k-2}+\vartheta \mathbf{e}_{n+k-2}^{\mathbf{q}}, \dot{\mathbf{q}}_{n+k-2}+\vartheta \mathbf{e}_{n+k-2}^{\dot{\mathbf{q}}}, \lambda_{n+k-2}+\vartheta \mathbf{e}_{n+k-2}^{\lambda}, t_{n+k-2}\right) \mathbf{e}_{n+k-2}^{\lambda} \mathrm{d} \vartheta \\
& +\mathcal{O}(1)\left(\left\|\mathbf{e}_{n+k-2}^{\mathbf{q}}\right\|+\left\|\mathbf{e}_{n+k-2}^{\dot{\mathbf{q}}}\right\|\right) \\
= & \mathbf{g}_{\lambda}\left(t_{n}\right) \mathbf{e}_{n+k-2}^{\lambda}+\mathcal{O}(h)\left\|\mathbf{e}_{n+k-2}^{\lambda}\right\|+\mathcal{O}(1)\left(\left\|\mathbf{e}_{n+k-2}^{\mathbf{q}}\right\|+\left\|\mathbf{e}_{n+k-2}^{\dot{\mathbf{q}}}\right\|\right)
\end{aligned}
$$

since $\mathbf{q}_{n+k-2}+\vartheta \mathbf{e}_{n+k-2}^{\mathbf{q}}=\mathbf{q}\left(t_{n+k-2}\right)+\mathcal{O}(h)=\mathbf{q}\left(t_{n}\right)+\mathcal{O}(h)$ etc., see (34). As before, the notation $\mathbf{g}_{\lambda}\left(t_{n}\right)$ is used as abbreviation for $\mathbf{g}_{\lambda}\left(\mathbf{q}\left(t_{n}\right), \dot{\mathbf{q}}\left(t_{n}\right), \boldsymbol{\lambda}\left(t_{n}\right), t_{n}\right)$.

The matrix product $\boldsymbol{\Phi}_{\mathbf{q}}\left(\mathbf{q}\left(t_{n}\right), t_{n}\right) \mathbf{g}_{\lambda}\left(t_{n}\right)$ is non-singular by the index-3 assumption, see (11-13). Therefore, the estimate for $\sum_{k} b_{k}^{\prime} \mathbf{e}_{n+k-2}^{\lambda}$ may be obtained multiplying (35) by $\left[\left(\boldsymbol{\Phi}_{\mathbf{q}} \mathbf{g}_{\lambda}\right)^{-1} \boldsymbol{\Phi}_{\mathbf{q}}\right]\left(\mathbf{q}\left(t_{n}\right), t_{n}\right)$ from the left and using the error recursion for $\mathbf{e}_{n}^{\mathbf{g}}$ from (30): 


$$
\begin{aligned}
\sum_{k=0}^{3} b_{k}^{\prime} \mathbf{e}_{n+k-2}^{\lambda}= & \left.\left(\boldsymbol{\Phi}_{\mathbf{q}} \mathbf{g}_{\lambda}\right)^{-1} \boldsymbol{\Phi}_{\mathbf{q}}\right]\left(\mathbf{q}\left(t_{n}\right), t_{n}\right) \cdot \sum_{k=0}^{3} b_{k}^{\prime} \mathbf{e}_{n+k-2}^{\mathbf{g}} \\
& +\mathcal{O}(1) \sum_{k=0}^{3}\left(\left\|\mathbf{e}_{n+k-2}^{\mathbf{q}}\right\|+\left\|\mathbf{e}_{n+k-2}^{\dot{\mathbf{q}}}\right\|+h\left\|\mathbf{e}_{n+k-2}^{\lambda}\right\|\right) \\
= & \mathcal{O}(1)\left\|\boldsymbol{\Phi}_{\mathbf{q}}\left(\mathbf{q}\left(t_{n}\right), t_{n}\right) \cdot \frac{1}{h} \sum_{k=0}^{2} a_{k} \mathbf{e}_{n+k-1}^{\mathbf{q}^{\prime}}\right\|+\mathcal{O}\left(\frac{1}{h}\right)\left\|\mathbf{l}_{n}^{\mathbf{q}^{\prime}}\right\| \\
& +\mathcal{O}(1) \sum_{k=0}^{3}\left(\left\|\mathbf{e}_{n+k-2}^{\mathbf{q}}\right\|+\left\|\mathbf{e}_{n+k-2}^{\dot{\mathbf{q}}}\right\|+h\left\|\mathbf{e}_{n+k-2}^{\lambda}\right\|\right) .
\end{aligned}
$$

With Lemma 3, see Sect. 4 below, we get

$$
\begin{aligned}
\sum_{k=0}^{3} b_{k}^{\prime} \mathbf{e}_{n+k-2}^{\lambda}= & \mathcal{O}\left(\frac{1}{h^{2}}\right) \sum_{k=0}^{3}\left\|\boldsymbol{\Phi}\left(\mathbf{q}_{n+k-2}, t_{n+k-2}\right)\right\|+\mathcal{O}\left(\frac{1}{h}\right)\left\|\mathbf{l}_{n}^{\mathbf{q}^{\prime}}\right\| \\
& +\mathcal{O}(1) \sum_{k=0}^{3}\left(\left\|\mathbf{e}_{n+k-2}^{\mathbf{q}}\right\|+\left\|\mathbf{e}_{n+k-2}^{\dot{\mathbf{q}}}\right\|+h\left\|\mathbf{e}_{n+k-2}^{\lambda}\right\|\right) \\
& +\mathcal{O}(1) \sum_{k=0}^{2}\left\|\mathbf{e}_{n+k-1}^{\mathbf{q}^{\prime}}\right\| .
\end{aligned}
$$

The equilibrium conditions at $t=t_{n+k-2}$ enforce $\boldsymbol{\Phi}\left(\mathbf{q}_{n+k-2}, t_{n+k-2}\right)=0$ and (37) may be summarized in the compact form

$$
\mathbf{e}_{n+1}^{\lambda}=-\frac{b_{0}^{\prime}}{b_{3}^{\prime}} \mathbf{e}_{n-2}^{\lambda}-\frac{b_{1}^{\prime}}{b_{3}^{\prime}} \mathbf{e}_{n-1}^{\lambda}-\frac{b_{2}^{\prime}}{b_{3}^{\prime}} \mathbf{e}_{n}^{\lambda}+\mathcal{O}(1) \sum_{k=0}^{3}\left\|\mathbf{e}_{n+k-2}^{\mathbf{v}}\right\|+\mathcal{O}(h) \sum_{k=0}^{3}\left\|\mathbf{e}_{n+k-2}^{\lambda}\right\|+\mathcal{O}\left(h^{2}\right) .
$$

The propagation of the errors $\mathbf{e}_{n}^{\lambda}$ is dominated by the recursion coefficients $b_{0}^{\prime}, b_{1}^{\prime}, b_{2}^{\prime}$ and $b_{3}^{\prime}$ and it is coupled with the errors $\mathbf{e}_{n}^{\mathbf{v}}$ in the differential components $\mathbf{q}, \dot{\mathbf{q}}, \mathbf{q}^{\prime}$ by $\mathcal{O}(1)$ coupling coefficients.

We note that the terms $\left\|\boldsymbol{\Phi}\left(\mathbf{q}_{n+k-2}, t_{n+k-2}\right)\right\| / h^{2}$ in (37) vanish in the formal convergence analysis, but may cause severe problems in a practical implementation of the method. Stopping the Newton iteration in Algorithm 1 with non-zero residuals $\mathbf{r}^{\mathbf{q}}, \mathbf{r}^{\lambda}$ may introduce small errors in $\left\|\boldsymbol{\Phi}\left(\mathbf{q}_{n+k-2}, t_{n+k-2}\right)\right\|$ that are amplified by the large factor $1 / h^{2}$ during time integration [1]. Therefore, the scaling method of Bottasso et al. [3] was used in the numerical tests of Sect. 5 to keep $\left\|\boldsymbol{\Phi}\left(\mathbf{q}_{n+k-2}, t_{n+k-2}\right)\right\|$ as small as possible, see also [13].

\subsection{Synthesis}

The error propagation in multistep methods may be studied in compact form writing the multistep method as one-step method in a higher dimensional configuration space [12].

With the error vectors

$$
\mathbf{E}_{n}^{\mathbf{v}}=\left(\begin{array}{c}
\mathbf{e}_{n}^{\mathbf{v}} \\
\mathbf{e}_{n-1}^{\mathbf{v}} \\
\mathbf{e}_{n-2}^{\mathbf{v}}
\end{array}\right), \quad \mathbf{E}_{n}^{\lambda}=\left(\begin{array}{c}
\mathbf{e}_{n}^{\lambda} \\
\mathbf{e}_{n-1}^{\lambda} \\
\mathbf{e}_{n-2}^{\lambda}
\end{array}\right)
$$


the propagation relations in (33) and (38) get the form

$$
\begin{aligned}
& \mathbf{E}_{n+1}^{\mathbf{v}}=\mathbf{A} \mathbf{E}_{n}^{\mathbf{v}}+\mathcal{O}(h)\left(\left\|\mathbf{E}_{n}^{\mathbf{v}}\right\|+\left\|\mathbf{E}_{n}^{\lambda}\right\|\right)+\mathcal{O}\left(h^{3}\right), \\
& \mathbf{E}_{n+1}^{\lambda}=\mathbf{B}^{\prime} \mathbf{E}_{n}^{\lambda}+\mathcal{O}(1)\left\|\mathbf{E}_{n}^{\mathbf{v}}\right\|+\mathcal{O}(h)\left\|\mathbf{E}_{n}^{\lambda}\right\|+\mathcal{O}\left(h^{2}\right)
\end{aligned}
$$

with error amplification matrices

$$
\mathbf{A}=\left(\begin{array}{ccc}
-\frac{a_{1}}{a_{2}} \mathbf{I} & -\frac{a_{0}}{a_{2}} \mathbf{I} & \mathbf{0} \\
\mathbf{I} & \mathbf{0} & \mathbf{0} \\
\mathbf{0} & \mathbf{I} & \mathbf{0}
\end{array}\right) \quad \text { and } \quad \mathbf{B}^{\prime}=\left(\begin{array}{ccc}
-\frac{b_{2}^{\prime}}{b_{3}^{\prime}} \mathbf{I} & -\frac{b_{1}^{\prime}}{b_{3}^{\prime}} \mathbf{I} & -\frac{b_{0}^{\prime}}{b_{3}^{\prime}} \mathbf{I} \\
\mathbf{I} & \mathbf{0} & \mathbf{0} \\
\mathbf{0} & \mathbf{I} & \mathbf{0}
\end{array}\right)
$$

In the unconstrained case, zero stability of the multistep method implies $\|\mathbf{A}\|=1$ in a suitable norm $\|$.$\| and second-order convergence follows by standard arguments from$ $\left\|\mathbf{E}_{n+1}^{\mathbf{v}}\right\| \leq(1+\mathcal{O}(h))\left\|\mathbf{E}_{n}^{\mathbf{v}}\right\|+\mathcal{O}\left(h^{3}\right)$, see [12].

In the constrained case, a similar argument is used to show that strict stability at infinity implies $\left\|\mathbf{B}^{\prime}\right\|<1$ in a (possibly different) norm $\|$.$\| for the algebraic solution compo-$ nents [13]. More precisely, the method is strictly stable at infinity if the roots $\zeta_{i}^{\prime}, i=1,2,3$, of the polynomial $\sigma$ in (22) are bounded by $\zeta_{\max }^{\prime}:=\max _{i}\left|\zeta_{i}^{\prime}\right|<1$, see Lemma 1 below, and $\left\|\mathbf{B}^{\prime}\right\|=\rho<1$ may be achieved for any $\rho>\zeta_{\max }^{\prime}$ using an appropriate norm $\|$.$\| for the alge-$ braic components [13].

Taking norms in (39) and (40), we obtain

$$
\left(\begin{array}{l}
\left\|\mathbf{E}_{n+1}^{\mathbf{v}}\right\| \\
\left\|\mathbf{E}_{n+1}^{\lambda}\right\|
\end{array}\right) \leq\left(\begin{array}{cc}
1+\mathcal{O}(h) & \mathcal{O}(h) \\
\mathcal{O}(1) & \rho+\mathcal{O}(h)
\end{array}\right)\left(\begin{array}{l}
\left\|\mathbf{E}_{n}^{\mathbf{v}}\right\| \\
\left\|\mathbf{E}_{n}^{\lambda}\right\|
\end{array}\right)+\left(\begin{array}{c}
\mathcal{O}\left(h^{3}\right) \\
\mathcal{O}\left(h^{2}\right)
\end{array}\right)
$$

and we deduce, as in [13], that the global errors after $n$ steps satisfy

$$
\left(\begin{array}{c}
\left\|\mathbf{E}_{n}^{\mathbf{v}}\right\| \\
\left\|\mathbf{E}_{n}^{\lambda}\right\|
\end{array}\right)=\mathcal{O}(1)\left\|\mathbf{E}_{0}^{\mathbf{v}}\right\|+\left(\mathcal{O}(h)+\mathcal{O}(1) \rho^{n}\right)\left\|\mathbf{E}_{0}^{\lambda}\right\|+\mathcal{O}\left(h^{2}\right) .
$$

Note, that strict stability at infinity allows to prove second-order convergence for all solution components despite the local error $\mathcal{O}\left(h^{2}\right)$ in the algebraic solution components, see (40). Furthermore, $\rho<1$ implies also that errors $\mathbf{E}_{0}^{\lambda}$ in the initial values of the Lagrange multipliers are damped out rapidly.

Summary In the constrained case, the generalized- $\alpha$ method with fixed time step-size $h$ has global errors $\mathcal{O}\left(h^{2}\right)$ in $\mathbf{q}$, $\dot{\mathbf{q}}$ and $\lambda$ if the order 2 condition in (20) and the stability conditions in (23) are satisfied and the errors $\mathbf{E}_{0}^{\mathbf{v}}, \mathbf{E}_{0}^{\lambda}$ in the initial values are $\mathcal{O}\left(h^{2}\right)$. The $\mathrm{CH}-\alpha$ algorithm with $\rho_{\infty} \in[0,1)$, see (24), may be considered as a typical example of a second-order convergent method for constrained systems.

\section{Technical details of the error estimates}

In the present section, some technical details of the convergence analysis are summarized. Readers, who are mainly interested in the basic steps of the analysis, may skip this section and continue with the results of numerical tests in Sect. 5 below. 
Lemma 1 A generalized- $\alpha$ scheme with parameters that satisfy the order 2 condition in (20) and the stability conditions in (23) is strictly stable at infinity, i.e., all roots $\zeta_{i}^{\prime}$ of polynomial $\sigma(\zeta)$, see (22), are inside the unit circle: $\left|\zeta_{i}^{\prime}\right|<1, i=1,2,3$.

Proof Polynomial $\sigma(\zeta)$ may be written as

$$
\sigma(\zeta)=\sum_{k=0}^{3} b_{k}^{\prime} \zeta^{k}=\beta\left(\left(1-\alpha_{f}\right) \zeta+\alpha_{f}\right)\left(\zeta^{2}+\frac{\frac{1}{2}+\gamma-2 \beta}{\beta} \zeta+\frac{\frac{1}{2}-\gamma+\beta}{\beta}\right)
$$

with roots

$$
\begin{aligned}
& \zeta_{1}^{\prime}=-\frac{\alpha_{f}}{1-\alpha_{f}}, \quad \zeta_{2,3}^{\prime}=-\frac{\frac{1}{2}+\gamma-2 \beta}{2 \beta} \pm \frac{R}{2 \beta} \quad \text { and } \\
& R:=\left(\left(\frac{1}{2}+\gamma-2 \beta\right)^{2}-4 \beta\left(\frac{1}{2}-\gamma+\beta\right)\right)^{1 / 2} .
\end{aligned}
$$

Because of $\alpha_{f}<1 / 2$ we get $\left|\zeta_{1}^{\prime}\right|<1$, see (23).

If the roots $\zeta_{2}^{\prime}, \zeta_{3}^{\prime}$ are complex, we have $\zeta_{2}^{\prime}=\bar{\zeta}_{3}^{\prime}$ and Vieta's Theorem implies

$$
\left|\zeta_{2}^{\prime}\right|=\left|\zeta_{3}^{\prime}\right|=\left(\zeta_{2}^{\prime} \zeta_{3}^{\prime}\right)^{1 / 2}=\left(\frac{\frac{1}{2}-\gamma+\beta}{\beta}\right)^{1 / 2}<\left(\frac{\beta}{\beta}\right)^{1 / 2}=1
$$

since $\alpha_{m}<\alpha_{f} \Rightarrow \gamma>1 / 2$, see (20).

For real roots $\zeta_{2}^{\prime}$, $\zeta_{3}^{\prime}$, we observe

$$
\begin{gathered}
R^{2}=\left(\frac{1}{2}+\gamma\right)^{2}-4 \beta<\left(\frac{1}{2}+\gamma\right)^{2} \Rightarrow R<\frac{1}{2}+\gamma \\
R^{2}=\left(4 \beta-\left(\frac{1}{2}+\gamma\right)\right)^{2}-8 \beta(2 \beta-\gamma)<\left(4 \beta-\left(\frac{1}{2}+\gamma\right)\right)^{2} \\
\Rightarrow-R>-\left(4 \beta-\left(\frac{1}{2}+\gamma\right)\right)
\end{gathered}
$$

since $2 \beta-\gamma>0$ and $4 \beta-\left(\frac{1}{2}+\gamma\right)=2\left(\beta-\frac{1}{4}\right)+(2 \beta-\gamma)>0$, see (20) and (23).

Therefore, real roots $\zeta_{2}^{\prime}$, $\zeta_{3}^{\prime}$ are bounded by

$$
\begin{aligned}
& \zeta_{2,3}^{\prime}<-\frac{\frac{1}{2}+\gamma-2 \beta}{2 \beta}+\frac{\frac{1}{2}+\gamma}{2 \beta}=\frac{2 \beta}{2 \beta}=1, \\
& \zeta_{2,3}^{\prime}>-\frac{\frac{1}{2}+\gamma-2 \beta}{2 \beta}-\frac{4 \beta-\left(\frac{1}{2}+\gamma\right)}{2 \beta}=-\frac{2 \beta}{2 \beta}=-1
\end{aligned}
$$

and $\left|\zeta_{i}^{\prime}\right|<1, i=1,2,3$ is guaranteed also in that case.

Lemma 2 For vectors $\mathbf{q}_{n+k-2}$ with $\left\|\mathbf{e}_{n+k-2}^{\mathbf{q}}\right\|=\mathcal{O}(h), k=0,1,2,3$, the terms $\boldsymbol{\Phi}_{\mathbf{q}}\left(\mathbf{q}\left(t_{n}\right), t_{n}\right) \mathbf{e}_{n+k-1}^{\mathbf{q}^{\prime}}, k=0,1,2$, satisfy (the arguments $t$ in $\boldsymbol{\Phi}$ and $\boldsymbol{\Phi}_{\mathbf{q}}$ are omitted for simplicity)

$$
\begin{aligned}
\boldsymbol{\Phi}_{\mathbf{q}}\left(\mathbf{q}\left(t_{n}\right)\right) \mathbf{e}_{n+k-1}^{\mathbf{q}^{\prime}}= & \frac{1}{h}\left(\boldsymbol{\Phi}\left(\mathbf{q}_{n+k-2}\right)-\boldsymbol{\Phi}\left(\mathbf{q}_{n+k-1}\right)\right)-\left.\frac{\partial}{\partial \mathbf{q}}\left(\boldsymbol{\Phi}_{\mathbf{q}}(\mathbf{q}) \mathbf{e}_{n+k-2}^{\mathbf{q}}\right)\right|_{\mathbf{q}=\mathbf{q}\left(t_{n}\right)} \cdot \dot{\mathbf{q}}\left(t_{n}\right) \\
& +\mathcal{O}(h)\left(\left\|\mathbf{e}_{n+k-1}^{\mathbf{q}}\right\|+\left\|\mathbf{e}_{n+k-2}^{\mathbf{q}}\right\|+\left\|\mathbf{e}_{n+k-1}^{\mathbf{q}^{\prime}}\right\|\right) \quad(h \rightarrow 0) .
\end{aligned}
$$


Proof To keep notation compact, the proof is given for $k=2$ and the argument $t$ in $\boldsymbol{\Phi}(\mathbf{q}, t)$ is omitted. The extension to $k=0,1$ and to $\boldsymbol{\Phi}=\boldsymbol{\Phi}(\mathbf{q}, t)$ is straightforward.

For linear time-invariant constraints $\boldsymbol{\Phi}(\mathbf{q}(t))=\mathbf{C q}(t)-\mathbf{z}=\mathbf{0}$, the proposition of the lemma is trivial since $\boldsymbol{\Phi}_{\mathbf{q}} \equiv \mathbf{C}, \boldsymbol{\Phi}_{\mathbf{q q}} \equiv \mathbf{0}$ and $\mathbf{e}_{n+1}^{\mathbf{q}^{\prime}}=\left(\mathbf{e}_{n+1}^{\mathbf{q}}-\mathbf{e}_{n}^{\mathbf{q}}\right) / h$, see (31):

$$
\mathbf{C} \mathbf{e}_{n+1}^{\mathbf{q}^{\prime}}=\mathbf{C}\left(\frac{\mathbf{q}\left(t_{n+1}\right)-\mathbf{q}\left(t_{n}\right)}{h}-\frac{\mathbf{q}_{n+1}-\mathbf{q}_{n}}{h}\right)=\frac{\mathbf{z}-\mathbf{z}}{h}-\frac{\left(\boldsymbol{\Phi}\left(\mathbf{q}_{n+1}\right)+\mathbf{z}\right)-\left(\boldsymbol{\Phi}\left(\mathbf{q}_{n}\right)+\mathbf{z}\right)}{h} .
$$

In the non-linear case, the identity

$$
-\boldsymbol{\Phi}\left(\mathbf{q}_{n}\right)=\boldsymbol{\Phi}\left(\mathbf{q}\left(t_{n}\right)\right)-\boldsymbol{\Phi}\left(\mathbf{q}_{n}\right)=\int_{0}^{1} \boldsymbol{\Phi}_{\mathbf{q}}\left(\mathbf{q}_{n}+\vartheta \mathbf{e}_{n}^{\mathbf{q}}\right) \mathbf{e}_{n}^{\mathbf{q}} \mathrm{d} \vartheta
$$

is used that follows from

$$
\boldsymbol{\psi}(1)-\boldsymbol{\psi}(0)=\int_{0}^{1} \boldsymbol{\psi}^{\prime}(\vartheta) \mathrm{d} \vartheta \quad \text { with } \quad \boldsymbol{\psi}(\vartheta):=\boldsymbol{\Phi}\left(\mathbf{q}_{n}+\vartheta \mathbf{e}_{n}^{\mathbf{q}}\right), \boldsymbol{\psi}^{\prime}(\vartheta)=\boldsymbol{\Phi}_{\mathbf{q}}\left(\mathbf{q}_{n}+\vartheta \mathbf{e}_{n}^{\mathbf{q}}\right) \mathbf{e}_{n}^{\mathbf{q}} .
$$

Because of (43), and the corresponding identity with $n$ being substituted by $n+1$, we have

$$
\begin{aligned}
\frac{1}{h}\left(\boldsymbol{\Phi}\left(\mathbf{q}_{n}\right)-\boldsymbol{\Phi}\left(\mathbf{q}_{n+1}\right)\right) \\
=\frac{1}{h} \int_{0}^{1} \boldsymbol{\Phi}_{\mathbf{q}}\left(\mathbf{q}_{n+1}+\vartheta \mathbf{e}_{n+1}^{\mathbf{q}}\right) \mathbf{e}_{n+1}^{\mathbf{q}} \mathrm{d} \vartheta-\frac{1}{h} \int_{0}^{1} \boldsymbol{\Phi}_{\mathbf{q}}\left(\mathbf{q}_{n}+\vartheta \mathbf{e}_{n}^{\mathbf{q}}\right) \mathbf{e}_{n}^{\mathbf{q}} \mathrm{d} \vartheta \\
=\int_{0}^{1} \boldsymbol{\Phi}_{\mathbf{q}}\left(\mathbf{q}_{n+1}+\vartheta \mathbf{e}_{n+1}^{\mathbf{q}}\right) \mathbf{e}_{n+1}^{\mathbf{q}^{\prime}} \mathrm{d} \vartheta \\
\quad+\frac{1}{h} \int_{0}^{1}\left(\boldsymbol{\Phi}_{\mathbf{q}}\left(\mathbf{q}_{n+1}+\vartheta \mathbf{e}_{n+1}^{\mathbf{q}}\right)-\boldsymbol{\Phi}_{\mathbf{q}}\left(\mathbf{q}_{n}+\vartheta \mathbf{e}_{n}^{\mathbf{q}}\right)\right) \mathbf{e}_{n}^{\mathbf{q}} \mathrm{d} \vartheta
\end{aligned}
$$

since $\left(\mathbf{e}_{n+1}^{\mathbf{q}}-\mathbf{e}_{n}^{\mathbf{q}}\right) / h=\mathbf{e}_{n+1}^{\mathbf{q}^{\prime}}$. The first term in the right-hand side of (44) may be written as

$$
\int_{0}^{1} \boldsymbol{\Phi}_{\mathbf{q}}\left(\mathbf{q}_{n+1}+\vartheta \mathbf{e}_{n+1}^{\mathbf{q}}\right) \mathbf{e}_{n+1}^{\mathbf{q}^{\prime}} \mathrm{d} \vartheta=\boldsymbol{\Phi}_{\mathbf{q}}\left(\mathbf{q}\left(t_{n}\right)\right) \mathbf{e}_{n+1}^{\mathbf{q}^{\prime}}+\mathcal{O}(h)\left\|\mathbf{e}_{n+1}^{\mathbf{q}^{\prime}}\right\|
$$

because $\mathbf{q}_{n+1}+\vartheta \mathbf{e}_{n+1}^{\mathbf{q}}=\mathbf{q}\left(t_{n+1}\right)+\mathcal{O}(h)=\mathbf{q}\left(t_{n}\right)+\mathcal{O}(h)$.

The second term in (44) contains curvature terms $\boldsymbol{\Phi}_{\mathbf{q q}}$. It vanishes in the linear timeinvariant case, but needs special care for non-linear constraints. The term may be expressed as

$$
\frac{1}{h} \int_{0}^{1}(\psi(1 ; \vartheta)-\psi(0 ; \vartheta)) \mathrm{d} \vartheta
$$

with $\boldsymbol{\psi}(\bar{\vartheta} ; \vartheta):=\boldsymbol{\Phi}_{\mathbf{q}}(\overline{\mathbf{q}}(\bar{\vartheta} ; \vartheta)) \mathbf{e}_{n}^{\mathbf{q}}$ and generalized coordinates

$$
\overline{\mathbf{q}}(\bar{\vartheta} ; \vartheta):=\mathbf{q}_{n}+\bar{\vartheta}\left(\mathbf{q}_{n+1}-\mathbf{q}_{n}\right)+\vartheta\left(\mathbf{e}_{n}^{\mathbf{q}}+\bar{\vartheta}\left(\mathbf{e}_{n+1}^{\mathbf{q}}-\mathbf{e}_{n}^{\mathbf{q}}\right)\right)=\mathbf{q}\left(t_{n}\right)+\mathcal{O}(h) .
$$

With these notations, we get in (46)

$$
\begin{gathered}
\frac{1}{h} \int_{0}^{1}(\boldsymbol{\psi}(1 ; \vartheta)-\boldsymbol{\psi}(0 ; \vartheta)) \mathrm{d} \vartheta=\frac{1}{h} \int_{0}^{1} \int_{0}^{1} \frac{\partial}{\partial \bar{\vartheta}} \boldsymbol{\psi}(\bar{\vartheta} ; \vartheta) \mathrm{d} \bar{\vartheta} \mathrm{d} \vartheta \\
\quad=\left.\frac{1}{h} \int_{0}^{1} \int_{0}^{1} \frac{\partial}{\partial \mathbf{q}}\left(\boldsymbol{\Phi}(\mathbf{q}) \mathbf{e}_{n}^{\mathbf{q}}\right)\right|_{\mathbf{q}=\overline{\mathbf{q}}(\bar{\vartheta} ; \vartheta)} \cdot \frac{\partial}{\partial \bar{\vartheta}} \overline{\mathbf{q}}(\bar{\vartheta} ; \vartheta) \mathrm{d} \bar{\vartheta} \mathrm{d} \vartheta
\end{gathered}
$$




$$
\begin{aligned}
= & \left.\int_{0}^{1} \int_{0}^{1} \frac{\partial}{\partial \mathbf{q}}\left(\mathbf{\Phi}(\mathbf{q}) \mathbf{e}_{n}^{\mathbf{q}}\right)\right|_{\mathbf{q}=\overline{\mathbf{q}}(\bar{\vartheta} ; \vartheta)} \cdot \dot{\mathbf{q}}\left(t_{n}\right) \mathrm{d} \bar{\vartheta} \mathrm{d} \vartheta+\mathcal{O}\left(\left\|\mathbf{e}_{n}^{\mathbf{q}}\right\|\right) \cdot \max _{\vartheta, \bar{\vartheta}}\left\|\frac{1}{h} \frac{\partial}{\partial \bar{\vartheta}} \overline{\mathbf{q}}(\bar{\vartheta} ; \vartheta)-\dot{\mathbf{q}}\left(t_{n}\right)\right\| \\
= & \left.\int_{0}^{1} \int_{0}^{1} \frac{\partial}{\partial \mathbf{q}}\left(\boldsymbol{\Phi}(\mathbf{q}) \mathbf{e}_{n}^{\mathbf{q}}\right)\right|_{\mathbf{q}=\mathbf{q}\left(t_{n}\right)} \cdot \dot{\mathbf{q}}\left(t_{n}\right) \mathrm{d} \bar{\vartheta} \mathrm{d} \vartheta+\mathcal{O}(h)\left\|\mathbf{e}_{n}^{\mathbf{q}}\right\| \\
& +\mathcal{O}\left(\left\|\mathbf{e}_{n}^{\mathbf{q}}\right\|\right) \cdot \max _{\vartheta, \bar{\vartheta}}\left\|\frac{\mathbf{q}\left(t_{n+1}\right)-\mathbf{q}\left(t_{n}\right)}{h}-(1-\vartheta) \mathbf{e}_{n+1}^{\mathbf{q}^{\prime}}-\dot{\mathbf{q}}\left(t_{n}\right)\right\| \\
= & \left.\frac{\partial}{\partial \mathbf{q}}\left(\boldsymbol{\Phi}(\mathbf{q}) \mathbf{e}_{n}^{\mathbf{q}}\right)\right|_{\mathbf{q}=\mathbf{q}\left(t_{n}\right)} \cdot \dot{\mathbf{q}}\left(t_{n}\right)+\mathcal{O}(h)\left\|\mathbf{e}_{n}^{\mathbf{q}}\right\|+\mathcal{O}(h)\left\|\mathbf{e}_{n+1}^{\mathbf{q}^{\prime}}\right\|
\end{aligned}
$$

since $\left\|\mathbf{e}_{n}^{\mathbf{q}}\right\|=\mathcal{O}(h)$ by assumption and $\mathbf{q}\left(t_{n+1}\right)-\mathbf{q}\left(t_{n}\right)=h \dot{\mathbf{q}}\left(t_{n}\right)+\mathcal{O}\left(h^{2}\right)$. The proof is completed substituting (45-47) in (44).

Lemma 3 With the assumptions of Lemma 2, the first term in the right-hand side of (36) satisfies (the arguments $t$ in $\boldsymbol{\Phi}$ and $\boldsymbol{\Phi}_{\mathbf{q}}$ are again omitted):

$$
\begin{aligned}
\boldsymbol{\Phi}_{\mathbf{q}}\left(\mathbf{q}\left(t_{n}\right)\right) \cdot \frac{1}{h} \sum_{k=0}^{2} a_{k} \mathbf{e}_{n+k-1}^{\mathbf{q}^{\prime}}= & \mathcal{O}\left(\frac{1}{h^{2}}\right) \sum_{k=0}^{3}\left\|\boldsymbol{\Phi}\left(\mathbf{q}_{n+k-2}\right)\right\| \\
& +\mathcal{O}(1)\left(\sum_{k=0}^{3}\left\|\mathbf{e}_{n+k-2}^{\mathbf{q}}\right\|+\sum_{k=0}^{2}\left\|\mathbf{e}_{n+k-1}^{\mathbf{q}^{\prime}}\right\|\right) .
\end{aligned}
$$

Proof The one-step nature of the generalized- $\alpha$ scheme results in a very special multistep representation of the error recursion in components $\mathbf{q}_{n}^{\prime}$ because $a_{1}=-1+2 \alpha_{m}=-(1-$ $\left.\alpha_{m}\right)+\alpha_{m}=-a_{2}-a_{0}$ :

$$
\frac{1}{h} \sum_{k=0}^{2} a_{k} \mathbf{e}_{n+k-1}^{\mathbf{q}^{\prime}}=\left(1-\alpha_{m}\right) \frac{\mathbf{e}_{n+1}^{\mathbf{q}^{\prime}}-\mathbf{e}_{n}^{\mathbf{q}^{\prime}}}{h}+\alpha_{m} \frac{\mathbf{e}_{n}^{\mathbf{q}^{\prime}}-\mathbf{e}_{n-1}^{\mathbf{q}^{\prime}}}{h} .
$$

Multiplying (49) by $\boldsymbol{\Phi}_{\mathbf{q}}\left(\mathbf{q}\left(t_{n}\right)\right)$ and applying Lemma 2 to

$$
\boldsymbol{\Phi}_{\mathbf{q}}\left(\mathbf{q}\left(t_{n}\right)\right) \frac{\mathbf{e}_{n+k-1}^{\mathbf{q}^{\prime}}-\mathbf{e}_{n+k-2}^{\mathbf{q}^{\prime}}}{h}=\frac{1}{h} \boldsymbol{\Phi}_{\mathbf{q}}\left(\mathbf{q}\left(t_{n}\right)\right) \mathbf{e}_{n+k-1}^{\mathbf{q}^{\prime}}-\frac{1}{h} \boldsymbol{\Phi}_{\mathbf{q}}\left(\mathbf{q}\left(t_{n}\right)\right) \mathbf{e}_{n+k-2}^{\mathbf{q}^{\prime}} \quad(k=1,2),
$$

we see that the estimate in (48) is a straightforward consequence of (49), Lemma 2 and

$$
\begin{aligned}
& -\left.\frac{\partial}{\partial \mathbf{q}}\left(\boldsymbol{\Phi}(\mathbf{q}) \frac{\mathbf{e}_{n+k-2}^{\mathbf{q}}-\mathbf{e}_{n+k-3}^{\mathbf{q}}}{h}\right)\right|_{\mathbf{q}=\mathbf{q}\left(t_{n}\right)} \cdot \dot{\mathbf{q}}\left(t_{n}\right)=-\left.\frac{\partial}{\partial \mathbf{q}}\left(\boldsymbol{\Phi}(\mathbf{q}) \mathbf{e}_{n+k-2}^{\mathbf{q}^{\prime}}\right)\right|_{\mathbf{q}=\mathbf{q}\left(t_{n}\right)} \cdot \dot{\mathbf{q}}\left(t_{n}\right) \\
& \quad=\mathcal{O}\left(\left\|\mathbf{e}_{n+k-2}^{\mathbf{q}^{\prime}}\right\|\right)
\end{aligned}
$$

for $k=1,2$.

\section{Numerical tests}

The following numerical tests have been developed in the formalism described by Géradin and Cardona [11], which allows to account for flexible bodies. Hence, the equations of motion are obtained in terms of absolute nodal coordinates with respect to the inertial frame. 
Fig. 1 Slider-crank mechanism
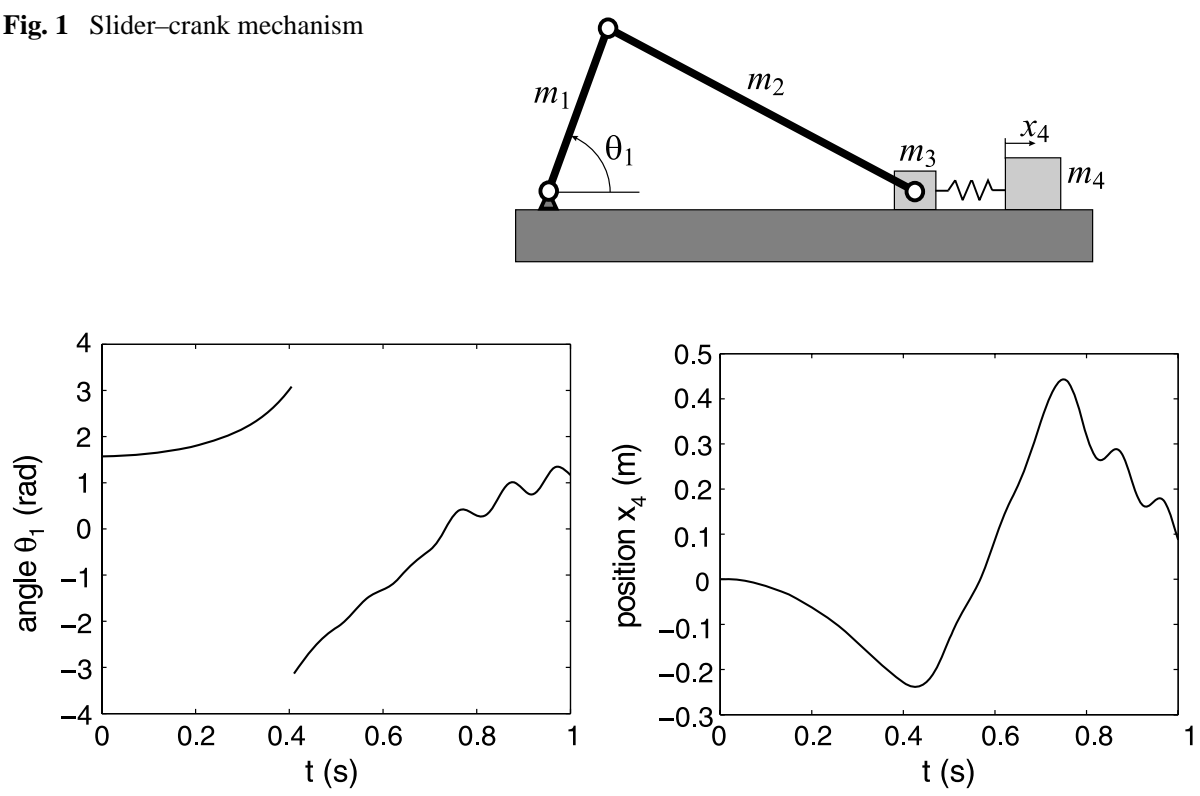

Fig. 2 Crank angle $\theta_{1}$ and position of the additional mass $x_{4}$

\subsection{Slider-crank mechanism}

The first example is a slider-crank mechanism with a spring-mass system attached to the sliding body (see Fig. 1). A similar benchmark has been considered in [16]. This planar system has two degrees-of-freedom that can be represented by the crank angle $\theta_{1}$ and the displacement of the additional mass $x_{4}$. The model involves 10 generalized coordinates: the positions of the centers of mass of body 1 and $2,\left(x_{1}, y_{1}, x_{2}, y_{2}\right)$, their orientation $\left(\theta_{1}, \theta_{2}\right)$, the position of bodies 3 and $4\left(x_{3}, x_{4}\right)$ and the position of the hinge connecting body 1 and 2 $\left(x_{5}, y_{5}\right)$. This set of coordinates has to satisfy 8 non-linear kinematic constraints.

The spring stiffness is $k=1000 \mathrm{~N} / \mathrm{m}$, the length of bodies 1 and 2 are $l_{1}=0.3 \mathrm{~m}$ and $l_{2}=0.6 \mathrm{~m}$, and the masses are $m_{1}=0.36 \mathrm{~kg}, m_{2}=0.15 \mathrm{~kg}, m_{3}=0.1 \mathrm{~kg}$ and $m_{4}=0.7 \mathrm{~kg}$. Initially, $\theta_{1}=\pi / 2$, the spring is undeformed and the mechanism is at rest. A constant torque $T=1 \mathrm{Nm}$ is applied to the crank and the initial conditions are computed so that the constraints are satisfied at position, velocity and acceleration level. The parameters of the generalized- $\alpha$ algorithm have been selected according to (24). The spectral radius of the algorithm is set to the typical value $\rho_{\infty}=0.7$, which leads to a sufficient amount of highfrequency numerical dissipation for the problem at hand. For a time step $h=5.0 \mathrm{e}-3 \mathrm{~s}$, the numerical results are given in Fig. 2.

A convergence study has been realized for $h \rightarrow 0$, and the results are plotted in Fig. 3 . The reference solution has been computed using a smaller time step. Second-order convergence is observed both for the generalized coordinates and the Lagrange multipliers.

\subsection{Andrews’ mechanism}

Andrews' squeezing mechanism, which is represented in Fig. 4, consists of seven articulated rigid bodies moving in a plane. This standard benchmark, described in details by 
Fig. 3 Convergence of relative errors at final time $(t=1 \mathrm{~s})$ for a generalized coordinate $\left(\theta_{1}\right)$ and a Lagrange multiplier $\left(\lambda_{1}\right)$

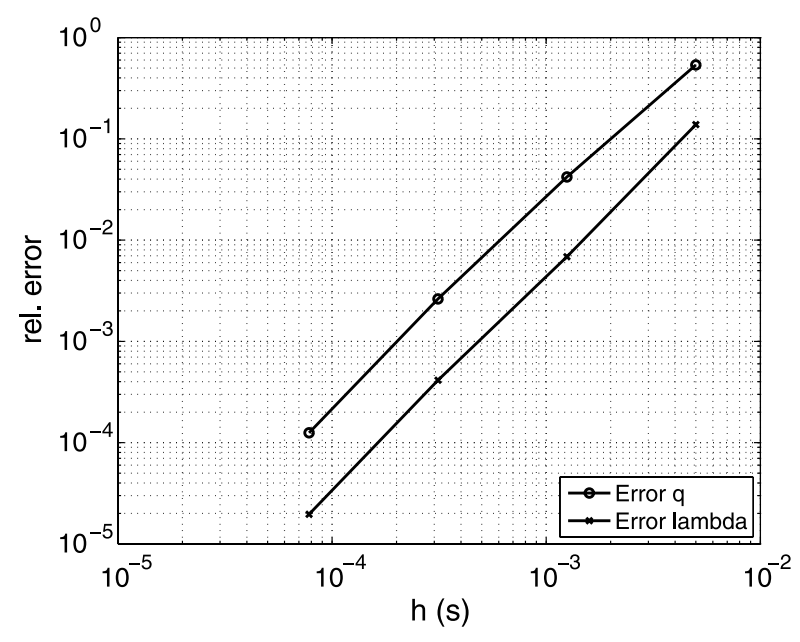

Fig. 4 Andrews' squeezing mechanism

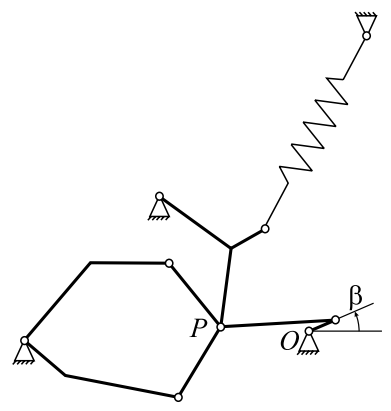

Schiehlen [19], has been largely exploited to demonstrate the performance of DAE timeintegration schemes. The mechanism has only one degree-of-freedom, and a constant torque is applied at point $O$. In the original benchmark, the equations of motion are explicitly given in terms of relative coordinates. In this work, one absolute rotation is defined for each body, whereas two translation coordinates are defined for each moving joint and each center of mass. Hence, the model involves a total of 31 generalized coordinates and 30 kinematic constraints. Initially, the mechanism is at rest, and the initial conditions are computed so that the constraints are satisfied at position, velocity and acceleration level.

As in the previous example, the parameters of the generalized- $\alpha$ algorithm have been selected according to (24) with a spectral radius $\rho_{\infty}=0.7$. For a time step $h=3.0 \mathrm{e}-4 \mathrm{~s}$, the numerical results are illustrated in Figs. 5, 6 and 7. Numerical damping is quite important to ensure a stable numerical solution, and a stable error propagation. For instance, Fig. 8 gives some results for the undamped algorithm $\left(\rho_{\infty}=1\right)$, which are strongly affected by numerical oscillations.

The results of a convergence analysis are plotted in Fig. 9. In the published benchmark, a reference solution is given for the body angles at final time. For the multipliers, we have computed a reference solution using a smaller time step. Second-order convergence is observed both for the generalized coordinates and the Lagrange multipliers. However, for very small $h$, the Lagrange multipliers are more sensitive to numerical errors. 

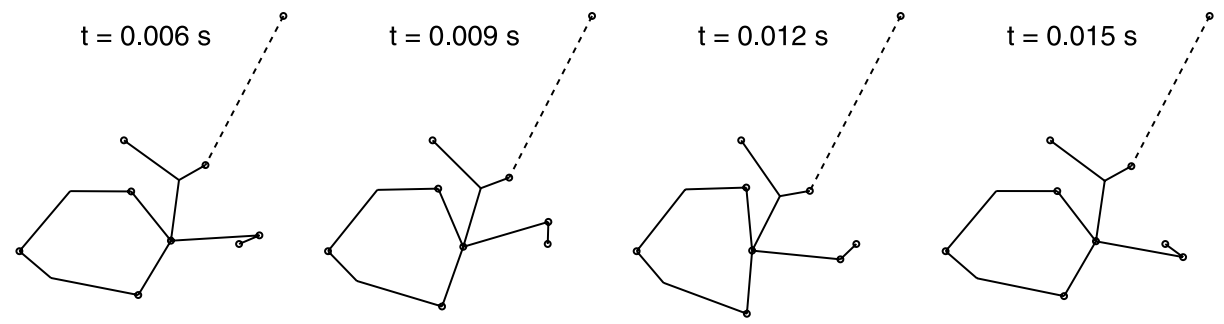

Fig. 5 Motion snapshots of the squeezing mechanism
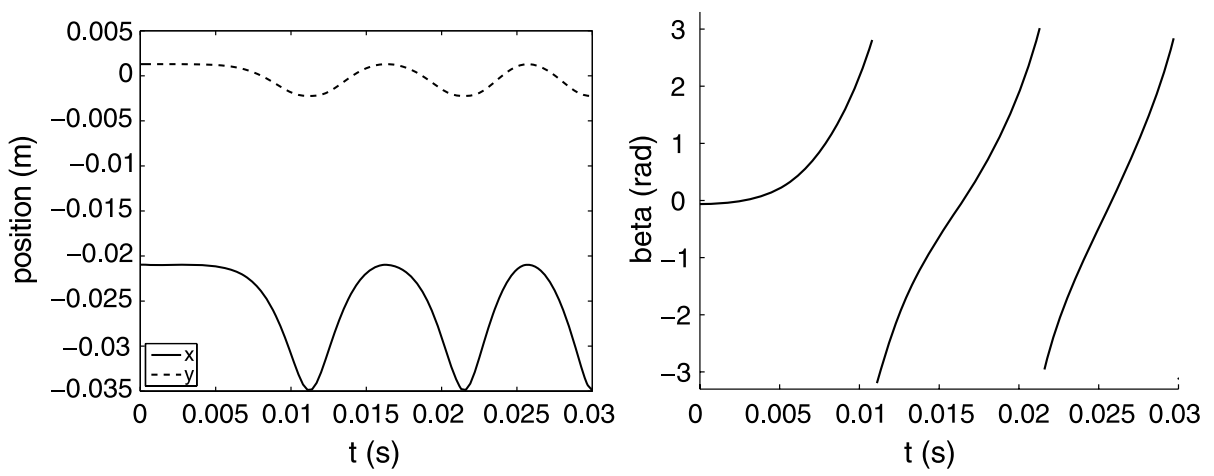

Fig. $6(x, y)$-position of point $P$ and angle $\beta$
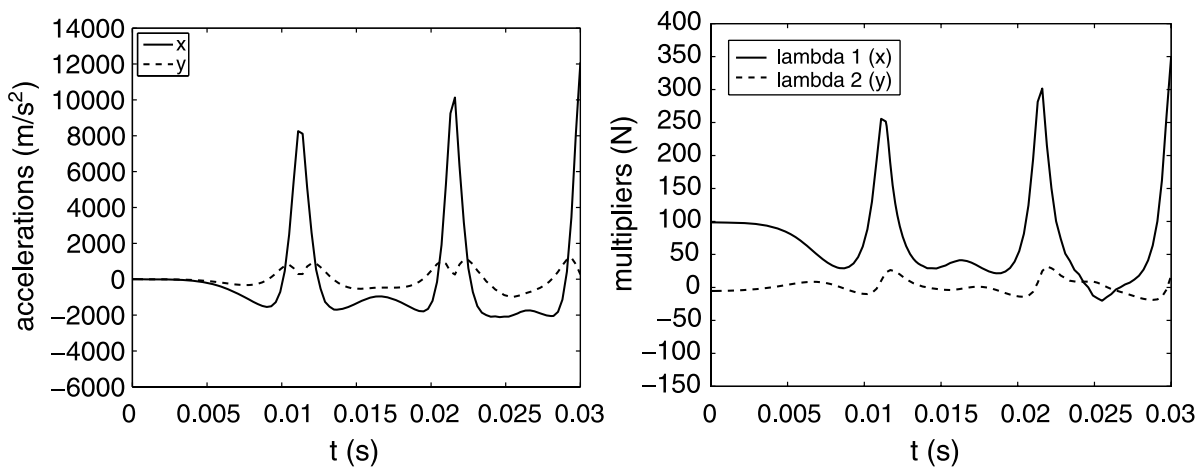

Fig. $7(x, y)$-accelerations of point $P$ and Lagrange multipliers

\section{Conclusions}

This paper analyses the accuracy of the generalized- $\alpha$ method for constrained mechanical systems. We note that the proposed algorithm, which is a variant of the original generalized- $\alpha$ algorithm, can deal with a non-constant mass matrix and that the accelerations are computed with second-order accuracy. 

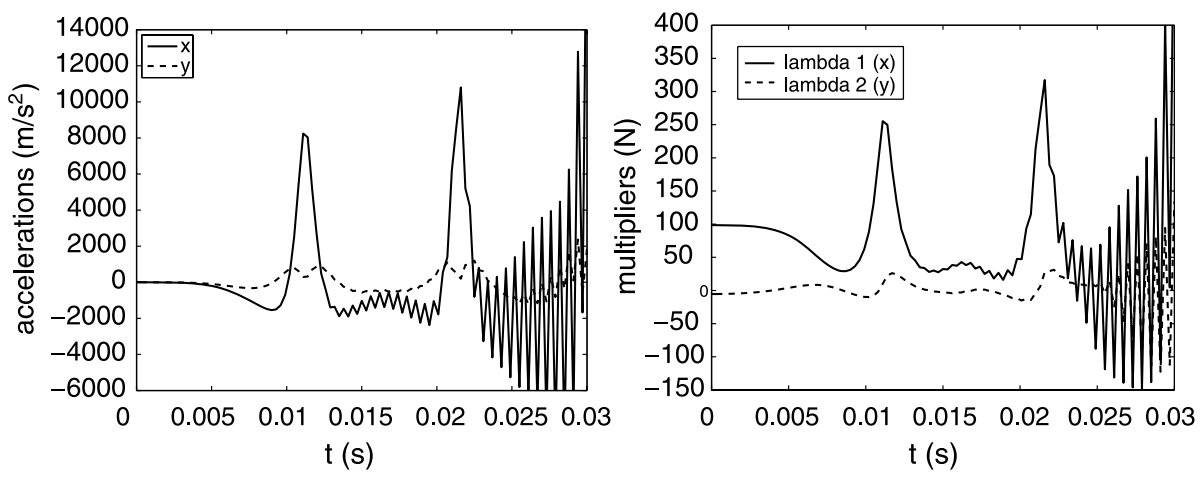

Fig. 8 Without numerical damping: $(x, y)$-accelerations of point $P$ and Lagrange multipliers associated with the $(x, y)$ internal forces in body 1

Fig. 9 Convergence of relative errors at final time $(t=0.03 \mathrm{~s})$ for a generalized coordinate $(\beta)$ and a Lagrange multiplier $\left(\lambda_{1}\right)$

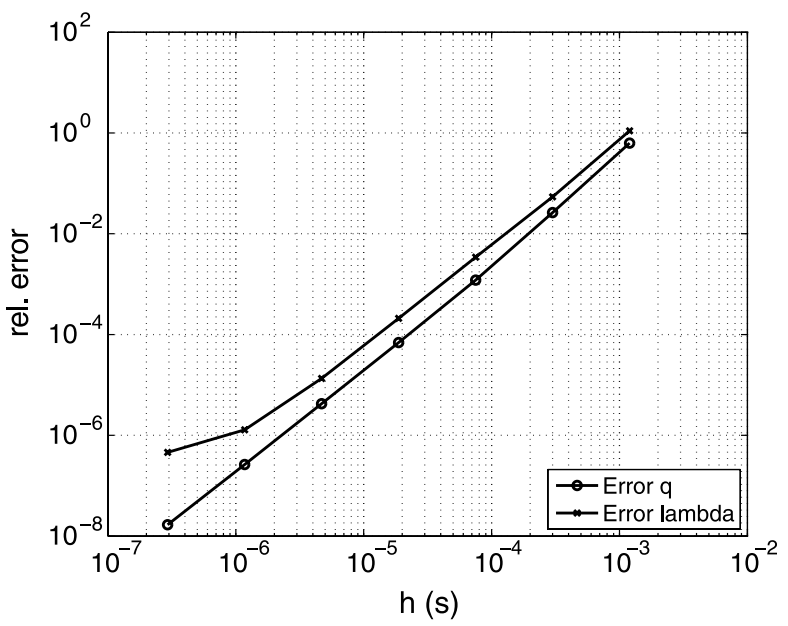

Using the analogy with multistep algorithms, global second-order convergence has been proven both for the generalized coordinates and the Lagrange multipliers. Those properties have been illustrated by numerical tests.

Acknowledgements $\mathrm{O}$. Brüls is supported by a grant from the Belgian National Fund for Scientific Research (FNRS) which is gratefully acknowledged. This work also presents research results of the Belgian Program on Inter-University Poles of Attraction initiated by the Belgian state, Prime Minister's office, Science Policy Programming. The scientific responsibility rests with its authors.

\section{References}

1. Arnold, M.: A perturbation analysis for the dynamical simulation of mechanical multibody systems. Appl. Numer. Math. 18, 37-56 (1995)

2. Arnold, M.: Simulation algorithms and software tools. In: Mastinu, G., Plöchl, M. (eds.) Road and Offroad Vehicle System Dynamics Handbook. Taylor \& Francis, London (2007, in preparation)

3. Bottasso, C., Dopico, D., Trainelli, L.: On the optimal scaling of index three DAEs in multibody dynamics. In: Proc. of the European Conference on Computational Mechanics (ECCOMAS-ECCM), Lisbon, Portugal (2006) 
4. Brenan, K., Campbell, S., Petzold, L.: Numerical Solution of Initial-Value Problems in DifferentialAlgebraic Equations, 2nd edn. SIAM, Philadelphia (1996)

5. Bruls, O., Arnold, M.: The generalized- $\alpha$ scheme as a multistep integrator: Towards a general mechatronic simulator. In: Proc. of the IDETC/MSNDC Conference, Las Vegas, USA (2007)

6. Bruls, O., Golinval, J.C.: The generalized- $\alpha$ method in mechatronic applications. Z. Angew. Math. Mech. (ZAMM) 86, 748-758 (2006)

7. Bruls, O., Golinval, J.C.: On the numerical damping of time integrators for coupled mechatronic systems. Comput. Meth. Appl. Mech. Eng. (2006), accepted for publication

8. Cardona, A., Géradin, M.: Time integration of the equations of motion in mechanism analysis. Comput. Struct. 33, 801-820 (1989)

9. Chung, J., Hulbert, G.: A time integration algorithm for structural dynamics with improved numerical dissipation: The generalized- $\alpha$ method. ASME J. Appl. Mech. 60, 371-375 (1993)

10. Erlicher, S., Bonaventura, L., Bursi, O.: The analysis of the generalized- $\alpha$ method for non-linear dynamic problems. Comput. Mech. 28, 83-104 (2002)

11. Géradin, M., Cardona, A.: Flexible Multibody Dynamics: A Finite Element Approach. Wiley, New York (2001)

12. Hairer, E., Norsett, S., Wanner, G.: Solving Ordinary Differential Equations I-Nonstiff Problems, 2nd edn. Springer, New York (1993)

13. Hairer, E., Wanner, G.: Solving Ordinary Differential Equations II-Stiff and Differential-Algebraic Problems, 2nd edn. Springer, New York (1996)

14. Hilber, H., Hughes, T., Taylor, R.: Improved numerical dissipation for time integration algorithms in structural dynamics. Earthq. Eng. Struct. Dyn. 5, 283-292 (1977)

15. Jay, L., Negrut, D.: Extensions of the HHT-method to differential-algebraic equations in mechanics. Electron. Trans. Numer. Anal. 26, 190-208 (2007)

16. Lunk, C., Simeon, B.: Solving constrained mechanical systems by the family of Newmark and $\alpha$-methods. Z. Angew. Math. Mech. (ZAMM) 86(10), 772-784 (2006)

17. Negrut, D., Rampalli, R., Ottarsson, G., Sajdak, A.: On the use of the HHT method in the context of index 3 differential algebraic equations of multi-body dynamics. In: Goicolea, J., Cuadrado, J., García Orden, J. (eds.) Proc. of the ECCOMAS Conf. on Advances in Computational Multibody Dynamics, Madrid, Spain (2005)

18. Newmark, N.: A method of computation for structural dynamics. ASCE J. Eng. Mech. Div. 85, 67-94 (1959)

19. Schiehlen, W. (ed.): Multibody Systems Handbook. Springer, Berlin (1990) 